\title{
Gadolinium-Enhanced Susceptibility-Weighted Imaging in Multiple Sclerosis: Optimizing the Recognition of Active Plaques for Different MR Imaging Sequences
}

\author{
(D)L.L.F. do Amaral, (DD.C. Fragoso, (D) R.H. Nunes, (DI.A. Littig, and (iD)A.J. da Rocha
}

\begin{abstract}
BACKGROUND AND PURPOSE: Gadolinium SWI is MR imaging that has recently been reported to be effective in the evaluation of several neurologic disorders, including demyelinating diseases. Our aim was to analyze the accuracy of gadolinium SWI for detecting the imaging evidence of active inflammation on MS plaques when a BBB dysfunction is demonstrated by a focal gadolinium-enhanced lesion and to compare this technique with gadolinium-enhanced $\mathrm{Tl}$ spin-echo and $\mathrm{Tl}$ spin-echo with magnetization transfer contrast.
\end{abstract}

MATERIALS AND METHODS: MR imaging studies of 103 patients (170 examinations) were performed using a 1.5T scanner. Two neuroradiologists scrutinized signal abnormalities of the demyelinating plaques on gadolinium SWI and compared them with gadolinium T1 before and after an additional magnetization transfer pulse. Interrater agreement was evaluated among gadolinium $\mathrm{Tl}$ magnetization transfer contrast, gadolinium SWI, and gadolinium $\mathrm{Tl}$ spin-echo using the $\kappa$ coefficient. The $\mathrm{Tl}$ magnetization transfer contrast sequence was adopted as the criterion standard in this cohort. Thus, the sensitivity, specificity, positive predictive value, and negative predictive value were calculated for gadolinium $\mathrm{Tl}$ spin-echo and gadolinium SWI sequences.

RESULTS: Differences in BBB dysfunction were evident among gadolinium SWI, gadolinium T1 spin-echo, and gadolinium T1 magnetization transfer contrast. Gadolinium $\mathrm{Tl}$ magnetization transfer contrast demonstrated the highest number of active demyelinating plaques. Gadolinium SWI was highly correlated with gadolinium T1 magnetization transfer contrast in depicting acute demyelinating plaques ( $\kappa$ coefficient $=0.860$; sensitivity $=0.837$ ), and these techniques provided better performance compared with gadolinium $\mathrm{T1}$ spin-echo $(\kappa$ coefficient $=0.78$; sensitivity $=0.645)$.

CONCLUSIONS: Gadolinium SWI was able to better detect BBB dysfunction in MS plaques and had a better performance than gadolinium T7 spin-echo. Increasing SWI sequence applications in clinical practice can improve our knowledge of MS, likely allowing the addition of BBB dysfunction analysis to the striking findings of the previously reported central vein sign.

ABBREVIATIONS: $\mathrm{Gd}=$ gadolinium; $\mathrm{MTC}=$ magnetization transfer contrast; $\mathrm{SE}=$ spin-echo

M ultiple sclerosis is a chronic and disabling demyelinating autoimmune disease that mainly affects young adults. MS is a multifactorial condition that probably results from the interaction among immunologic, genetic, and environmental factors, ${ }^{1}$

Received December 6, 2018; accepted after revision January 24, 2019.

From the Division of Neuroradiology (L.L.F.A, D.C.F., R.H.N., I.A.L., A.J.R.), Faculdade de Ciências Médicas da Santa Casa de São Paulo, São Paulo, Brazil; Neuroradiology Department (L.L.F.A.), BP Medicina Diagnóstica, Hospital BP e BP Mirante da Beneficēncia Portuguesa de São Paulo, São Paulo, Brazil; Division of Neuroradiology (D.C.F., R.H.N., A.J.R.), Diagnósticos da América S.A. - DASA, São Paulo, Brazil; and Division of Neuroradiology (D.C.F.), Fleury S.A., São Paulo, Brazil.

L.L.F.A was supported by Coordenação de Aperfeiçoamento de Pessoal de Nível Superior (CAPES), which partially supported this research.

Please address correspondence to Lázaro Luís Faria do Amaral, MD, Rua Dr. Cesario Mota Junior, 112, vila buarque, 01221-020, São Paulo, São Paulo, Brazil; e-mail: neuro.medimagem@gmail.com

- Indicates open access to non-subscribers at www.ajnr.org

http://dx.doi.org/10.3174/ajnr.A5997 predominantly characterized by the independent processes of inflammation, demyelination, neurodegeneration, remyelination, and axonal repair in different multifocal combinations at different stages of disease progression. MS is one of the most common neurologic disorders in the world and, in many countries, is the leading cause of nontraumatic neurologic disability in young adults. ${ }^{2}$

MR imaging produces contrasts according to different tissues. Combined with its noninvasiveness, MR imaging has become the technique of choice for in vivo imaging of several disorders. Currently, MR imaging is used in all MS work-up processes ${ }^{3}$ because it facilitates earlier diagnosis, helps to safely exclude potential differential diagnoses, and contributes to the evaluation of disease activity and potential complications linked to the treatment. ${ }^{3,4}$

Currently, diagnosis of MS is fundamentally based on demonstrating the dissemination both in time and in space of the demyelinating process, excluding mimicking disorders (no better ex- 
planation). ${ }^{5}$ Although diagnosis of MS mainly consists of the clinical criteria attributable to this process, imaging has become the most important paraclinical tool because it can corroborate or even refute a clinical suspicion, ${ }^{3}$ especially in cases in which demyelinating plaques involve noneloquent areas with little or no expression (clinical-radiologic paradox). ${ }^{6}$

Magnetic Resonance Imaging in MS (MAGNIMS; https:// www.magnims.eu/) emphasizes the role of MR imaging in MS evaluations ${ }^{7}$ and indicates a need for an initial examination (first 6 months) and follow-up examinations every 12 months to assess the presence of new $\mathrm{T} 2$ lesions and to search for foci of active inflammation using the postgadolinium T1 sequence. Gadolinium (Gd) enhancement of MS plaques is a well-established marker of MS lesion inflammation. ${ }^{8}$ This biomarker has been mainly used in the diagnosis and monitoring of patients with MS because contrastenhancing lesions reflect BBB dysfunction and the inflammatory response. ${ }^{9}$ Additionally, recent studies have shown the prognostic role and clinical correlation of Gd-enhancing lesions as an independent long-term predictor in clinically isolated syndrome. ${ }^{10}$

Susceptibility-weighted imaging is a relatively new MR imaging technique that is currently being implemented in clinical practice in addition to conventional spin density and T1- and T2-weighted imaging methods, which are already used in commercially available MR imaging scanners. A number of studies have investigated a central vein inside white matter lesions, the "central vein sign," detected by SWI or $2^{\star}$-weighted magnitude images, as a promising imaging biomarker of inflammatory demyelination, adding MS specificity to the diagnosis. ${ }^{11}$ To standardize the evaluation of the central vein sign in the diagnosis of MS, the North American Imaging in Multiple Sclerosis group provided statements and recommendations. ${ }^{12} \mathrm{Al}-$ though 7T has demonstrated the highest sensitivity for central vein detection, ${ }^{13} 1.5 \mathrm{~T}$ has also shown high rates of central vein sign detection. ${ }^{14}$ A finding of nonconfluent lesions $3 \mathrm{~mm}$ in length with 1 central vein has been demonstrated as a sensitive and specific discriminator of patients with relapsing-remitting MS from control subjects with benign white matter lesions. A study conducted by Hosseini et $\mathrm{al}^{13}$ also demonstrated a hypointense rim around MS lesions as an adjunct imaging biomarker for MS, which may be used with the central vein sign as a radiologic sign to differentiate benign from MS white matter lesions.

Additionally, to provide information about any tissue that has a different susceptibility than its surrounding structures, such as deoxygenated blood, hemosiderin, ferritin, and calcium (which are revealed by the $\mathrm{T} 2{ }^{*}$ effect), ${ }^{15}$ this technique also allows the $\mathrm{T} 2$ effect to be analyzed-that is, the effect for brain edema; with an appropriate pulse angle, this approach can also take advantage of tissue longitudinal relaxation time analysis. A recent report also argued that under certain circumstances or for certain disorders, the T1-relaxivity may dominate and a T1 shinethrough phenomenon may be observed. ${ }^{16}$

The applicability of the T1-shinethrough effect might be pivotal for exploring the characteristics of Gd-based contrast agents on SWI, which primarily have a T1-shortening effect, ${ }^{17}$ without compromising the imaging of the normal brain. ${ }^{18}$ On the basis of this background, our study was designed to investigate the accuracy of the Gd-SWI sequence to detect BBB dysfunctions in MS plaques in the brain by comparing it with the performance of sequences that are currently used in clinical practice.

\section{MATERIALS AND METHODS \\ Standard Protocol Approvals}

The study was approved by an institutional review board of Santa Casa de São Paulo, and written informed consent was obtained from all participants.

\section{Study Design and Subjects}

This study is an observational retrospective analysis of consecutive brain MR imaging examinations from a series of patients with a defined MS diagnosis. MR images were accessed through the digital archive of the institution and were acquired from December 2013 to December 2016. All patients had imaging findings indicative of MS according to the 2017 revised McDonald Criteria. ${ }^{5}$ Studies that did not fulfill the correct institution protocol, had poor-quality images that limited interpretation, had images with an alternative diagnosis that supported the symptoms, and had discrepancies in parameters that might affect the interpretation and comparison of images were all excluded from the study.

\section{Data Acquisition}

All data were acquired on a 1.5T scanner (Achieva; Philips Healthcare, Best, the Netherlands) using a 16-channel sensitivity encoding neurovascular coil. Sagittal volumetric FLAIR images were acquired for brain lesion detection (slice thickness, $0.7 \mathrm{~mm}$; FOV, $220 \times 220 \times 180 \mathrm{~mm}^{3}$; matrix, $184 \times 184$; TR/TE/TI, 7.000/263/ $2.300 \mathrm{~ms}$; and acquisition time, 8 minutes and 31 seconds).

Gd-SWI data were acquired with a flow-compensated 3D gradient-echo method (FOV, $220 \times 189 \times 150 \mathrm{~mm}^{3}$; matrix, $220 \times 189$; voxel size, $1.0 \times 1.0 \times 1.0 \mathrm{~mm}^{3}$; TR/TE, $23 / 33 \mathrm{~ms}$; flip angle $10^{\circ}$; slice thickness, $1 \mathrm{~mm}$; acquisition time, 3 minutes and 20 seconds).

The 2D-T1 spin-echo (SE) sequence was acquired using the following parameters: 25 slices; slice thickness, $5 \mathrm{~mm}$; FOV, $220 \times$ $189 \times 126 \mathrm{~mm}^{3}$; matrix, $244 \times 168$; TR/TE, $614 / 15 \mathrm{~ms}$; acquisition time, 1 minute and 45 seconds. A comparative 2D-T1 SE sequence with an additional magnetization transfer contrast (MTC) on-resonance pulse (25 slices; slice thickness, $5 \mathrm{~mm}$; gap, $0.5 \mathrm{~mm}$; FOV , $220 \times 200 \times 137 \mathrm{~mm}^{3}$; matrix, $212 \times 134$; TR/TE, $600 / 12 \mathrm{~ms}$; and acquisition time, 5 minutes and 26 seconds) was also obtained before and after intravenous administration of 0.1 $\mathrm{mmol} / \mathrm{kg}$ of Gd (Gadovist [gadobutrol]; Bayer Schering Pharma, Berlin, Germany).

The order of acquisition of the postcontrast sequences was uniform in all studies. The SWI sequence was acquired first, followed by the T1 SE, and finally, the T1 MTC.

\section{Imaging Analysis}

All images were assessed by 2 experienced neuroradiologists (A.J.d.R. and L.L.F.d.A.) with 18 and 25 years of neuroimaging experience who were blinded to the patient identity and clinical data to avoid any recall bias. The observers separately analyzed Gd-SWI, Gd-T1 SE, and Gd-T1 MTC and always evaluated the results in that order. The precontrast FLAIR sequence was always assessed to the interpretation to confirm the lesion features. Strict criteria were applied to designate a lesion as enhanced on Gd- 


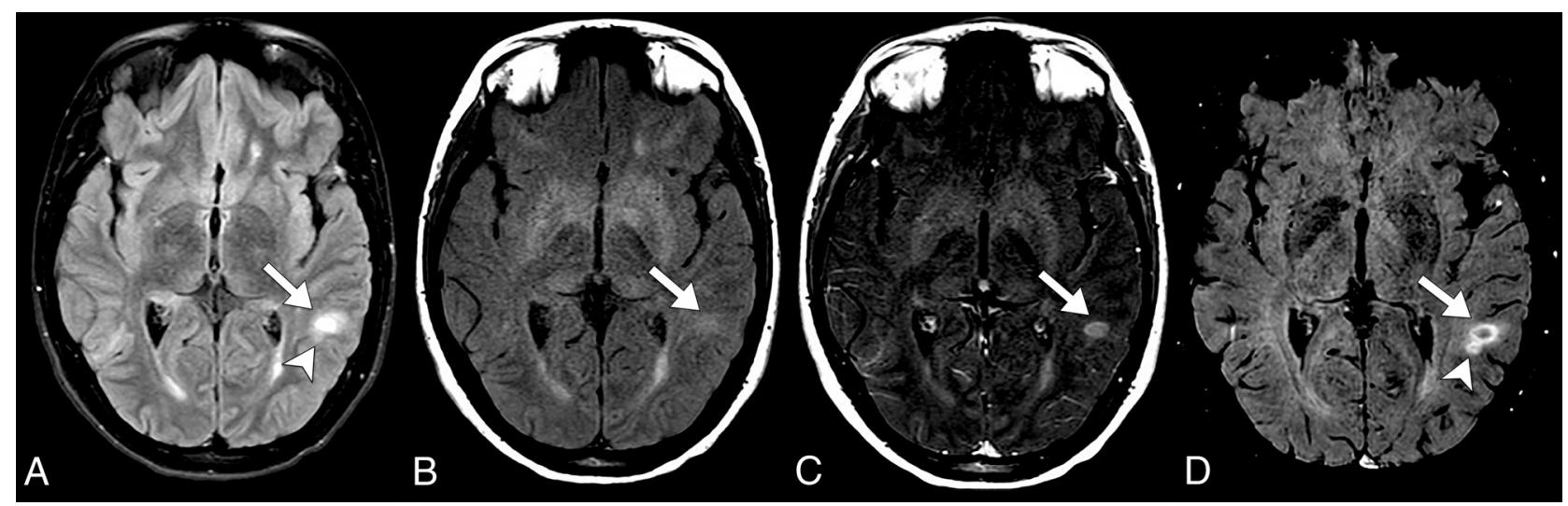

FIG 1. Axial fluid-attenuated inversion recovery sequence $(A)$, axial T1 MTC pre- and postcontrast ( $B$ and $C$ ), and axial Gd-SWI sequence $(D)$. A definitive acute demyelinating lesion requires the demonstration of a Gd-enhancing lesion along with high signal in the fluid-sensitive sequences, as shown in the left subcortical white matter temporal gyrus lesion (arrows). Note a small ovoid high-signal lesion on a FLAIR sequence adjacent to the aforementioned lesion, which demonstrates BBB dysfunction that is only characterized by the Gd-SWI sequence (arrowheads).

Table 1: Active plaque characteristics ${ }^{\mathrm{a}}$

\begin{tabular}{|c|c|c|c|c|c|c|}
\hline \multicolumn{3}{|c|}{ Distribution } & \multicolumn{4}{|c|}{ Morphology } \\
\hline Subcortical & Periventricular & Posterior Fossa & Nodular & Annular & Tumefactive & Punctate \\
\hline 50.8 & 45.3 & 3.9 & 68.8 & 23.1 & 10.2 & 21.2 \\
\hline
\end{tabular}

${ }^{a}$ Spatial distribution and morphology of the Gd-enhancing lesions.

SWI; all definite enhancing lesions were included (Fig 1), whereas areas of bright signal that were indistinguishable from flow artifacts or Gd-DTPA contrast within vessels or that did not have a comparable high signal on FLAIR were excluded.

The readers were asked to separate Gd-enhancing lesions according to their location in subcortical/juxtacortical, periventricular, or infratentorial (posterior fossa) areas, and the Gd-enhancing pattern was determined to be nodular, annular, punctate, or tumefactive. ${ }^{19}$ Similarly, we separately performed a comparative analysis of the interpretation of both Gd-T1 SE and Gd-T1 MTC to search for either a similar Gd enhancement in SWI lesions or a Gd-SWI acquisition that was negative for the presence of lesions enhancing in the other sequences.

The Gd-T1 MTC sequence was considered the criterion standard tool for determining lesions positive for acute plaques because it is considered the preferred method in a $1.5 \mathrm{~T}$ scanner. ${ }^{20-22}$

\section{Statistical Analyses}

Statistical analysis was performed using the Statistical Package for the Social Sciences, Version 21 (IBM, Armonk, New York).

Interrater agreement for Gd enhancement among postcontrast sequences was assessed with the Cohen $\kappa$ coefficient. Agreement was graded according to the Altman definition with $0.0-$ 0.20 , poor; $0.21-0.40$, fair; $0.41-0.60$, moderate; $0.61-0.80$, good; and $0.81-1.00$, very good.

According to the current design, the sensitivity, specificity, positive predictive value, and negative predictive value were calculated for Gd-T1 SE and Gd-SWI sequences to depict active demyelinating lesion-detectability performance.

\section{RESULTS}

According to the defined criteria, a total of 170 examinations were included in this cohort. The study population included 103 pa-
Table 2: Frequency characteristics

\begin{tabular}{lcccc}
\hline Sequence & Sensitivity & Specificity & PPV & NPV \\
\hline Gd-SWI & 0.837 & 0.998 & 0.891 & 0.977 \\
Gd-TI SE & 0.645 & 1 & 0.995 & 0.994 \\
\hline
\end{tabular}

Note:-PPV indicates positive predictive value; NPV, negative predictive value.

tients with relapsing-remitting MS (76 women; mean age, $36.4 \pm$ 11.6 years; range, $16-63$ years).

A total of 15,756 white matter lesions (range, 2-400 lesions per patient) were detected with a FLAIR sequence. The Gd-T1 MTC sequence showed the highest number of lesions with Gd enhancement with 282 lesions, followed by Gd-SWI with 265 lesions, and finally, Gd-T1 SE with 183 lesions. The spatial distribution and morphology of the Gd-enhancing lesions are shown in Table 1.

Very good interrater agreement was observed for Gd-enhancing lesion detection between the Gd-SWI and Gd-T1 MTC sequences ( $\kappa$ coefficient $=0.86$ ). Although 46 lesions were erroneously interpreted as negative for Gd-enhancement, 29 lesions were only demonstrated in the SWI sequence.

Although moderate interrater agreement was observed between Gd-T1 SE and Gd-T1 MTC $(\kappa$ coefficient $=0.78), 100$ lesions were missed by the SE acquisition.

If one assumes that Gd-T1 MTC was the criterion standard sequence, the Gd-SWI and Gd-T1 SE frequency characteristics in this cohort are demonstrated in Table 2.

When the Gd-T1 SE and Gd-SWI sequence results were compared, we observed that 82 more lesions were detected by the SWI acquisition, which resulted in a sensitivity increase of approximately $29.7 \%$.

Considering the different lesion morphologies, larger lesions $(>2.0 \mathrm{~cm})$ had the highest agreement rate $(99.3 \%)$. Conversely, punctate lesions demonstrated a lower agreement, but there was still a substantial concordance rate (91.0\%) among all the post-Gd sequences that were analyzed. 


\section{DISCUSSION}

A considerable increase in the physiopathogenic mechanisms of MS was achieved with the advent of MR imaging from its debut in the 1970s until today. MS has long been regarded as a chronic inflammatory disease of the white matter that leads to demyelination and, eventually, to progressive neurodegeneration. The pathogenesis of MS is fundamentally characterized by 2 distinctbut-intermingled processes ${ }^{23,24}$ : inflammation, which predominantly occurs in the early stages of the demyelinating process, and neurodegeneration, which is presumably dominant in the later stages of the disease but also begins in the early stages. ${ }^{25}$

Although activity and progression are very close to one another, in clinical practice, their analysis is approached separately. Disease activity is defined by clinical relapses and/or lesion activity in CNS imaging and is related to episodes of tissue damage associated with inflammation. ${ }^{26}$ Progression is linked to increasing neurologic dysfunction, which (by current understanding) reflects neurodegenerative processes. ${ }^{27}$ According to the current criteria, ${ }^{5}$ analysis of the therapeutic evaluation based on the estimation of active disease is performed by MR imaging determining the lesion load (ie, the number of new lesions or increase in the volume of pre-existing lesions) and detecting the BBB dysfunction represented by Gd enhancement. ${ }^{7}$

Gd-based contrast agents used in conjunction with MR imaging have been approved by regulatory agencies around the world. Several studies have shown their efficacy for improving the accuracy of MR imaging studies, and they currently have a positive cumulative safety record. ${ }^{28}$ Nevertheless, concerns have arisen about the long-term health effects of repeat Gd administration in patients with MS and the incomplete characterization of the MS lesion pathophysiology that results from relying on contrast-enhancement characteristics alone. Investigations have shown evidence of Gd deposition within the human brain after multiple Gd contrast administrations, especially in the dentate nucleus and globus pallidus, particularly when linear compounds are used. ${ }^{29}$ Thus, the National Institutes of Health recommends that the necessity of Gd administration in specific clinical indications should be carefully re-evaluated given the uncertain long-term public health impact of the deposition of Gd within the brain, ${ }^{28}$ though the precise causal role, if any, that repeat Gd injections play in MS pathogenesis remains unknown.

Increasing investigations have focused on the search for biomarkers that ideally demonstrate early inflammation and neurodegeneration processes separately, which would allow a more confident analysis of the therapeutic response and prognostic analysis at the individual level. ${ }^{30}$

Promising alternatives in determining the MS neuroinflammation and excitotoxicity processes in vivo have been demonstrated. It has been documented in the literature and adopted by our institution that the T1 MTC using 1.5T equipment is the most sensitive sequence for the demonstration of a BBB disruption with inflammatory activity in MS. ${ }^{20-22,31}$ Nevertheless, there have been some controversies in the literature on this subject, and the lack of consistency among these methods for different MR imaging scanners is the main debate.

Potential sequences that also require Gd-based contrast that have been recently reported in the literature are the double inver- sion recovery ${ }^{32}$ and T1 3D MPRAGE. ${ }^{33}$ Nonetheless, some investigations have also evaluated the accuracy of noncontrast MR imaging to confidently detect acute MS plaques. ${ }^{34}$ Diffusion tensor imaging, particularly through the fractional anisotropy metric, ${ }^{35}$ and, more recently, analysis of quantitative susceptibility mapping $^{36}$ have been demonstrated to have high diagnostic accuracy for distinguishing enhancing from nonenhancing MS plaque activity in the absence of Gd administration. It is also worth mentioning sequences that label antibodies targeting surface markers of immune cells in preclinical studies. ${ }^{37}$ These imaging biomarkers may also have additional benefits for providing insight into the MS pathobiology that is not possible due to the simple dichotomous characterization of the presence or absence of contrast enhancement, and which could increase the accuracy of MS diagnosis, improve disease prognostication, and provide a more robust marker of treatment response.

SWI is a fully velocity-compensated, long-echo, radiofrequency-spoiled, high-resolution, 3D gradient-echo sequence that combines magnitude and filtered-phase information to generate clinical information in addition to anatomic images. ${ }^{38}$ Since its introduction in the beginning of this century, ${ }^{39}$ a substantial increase in information has been achieved through magnetic susceptibility sequences. ${ }^{15}$ SWI takes advantage of the susceptibility differences among tissues, which leads to phase differences (phase) and causes a signal loss (magnitude). ${ }^{39}$ In clinical practice, SWI has added to the recognition of small calcifications, parenchymal microbleeds, cavernous malformations, and traumatic shearing injuries. ${ }^{17}$ SWI also allows visualization of small vessels and veins within the brain, which is an advantage used for venous thrombosis, arteriovenous malformations, dural arteriovenous fistulas, and stroke, ${ }^{15}$ in addition to revealing a vein within a demyelinating lesion (central vein sign), translating the perivenular demyelination, and increasing the specificity and pathologic correlation of the image findings. ${ }^{40}$

SWI is routinely performed without intravenous Gd administration. Nevertheless, no image-quality degradation and no significant signal intensity changes in the gray matter and WM have been recognized after intravenous Gd administration. ${ }^{18}$ The magnitude signal intensity depends on $\mathrm{T}^{*}$, geometry, orientation to the $\mathrm{B}_{0}$, and differences in magnetic susceptibility, as well as serving as a function of $\mathrm{T} 1$. Thus, although $\mathrm{T} 2^{\star}$ and phase effects are dominant, there is still a T1-relaxivity term present when the flip angle is greater than or close to the Ernst angle $[\theta \mathrm{E}=$ square $\operatorname{root}(2 \mathrm{TR} / \mathrm{T} 1)] .^{16}$

In certain settings, the T1-relaxivity may dominate and the T1-shinethrough phenomenon may arise, such as the effects that occur in the cerebral venous sinuses after Gd administration, which typically measure a few centimeters in diameter. ${ }^{18}$ Through this principle, the evaluation of Gd leakage due to dysfunction of the BBB has been exploited with some benefits and promising results in several situations, especially in the field of neuro-oncologic imaging, ${ }^{41}$ as well as in the evaluation of inflammatory disorders, as shown in this study for MS inflammation-activity detection.

Our results support the use of the post-Gd SWI sequence in assessing inflammatory activity in patients with MS (Fig 2) and show that pre- and post-Gd SWI demonstrate a diagnostic 


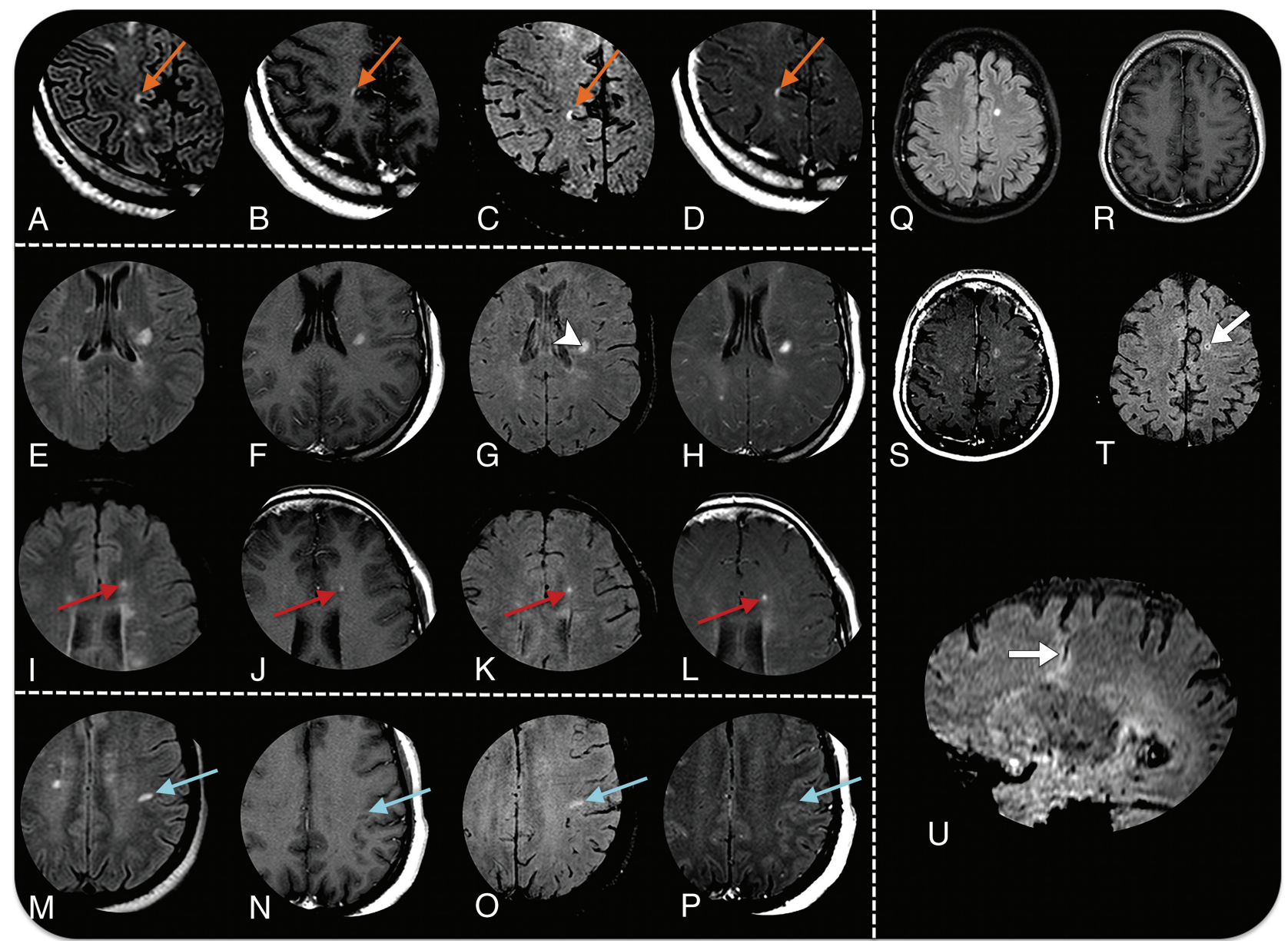

FIG 2. MR imaging detection of active plaques in 4 different patients with $M S$. Axial fluid-attenuated inversion recovery sequences $(A, E, I, M$, and $Q$ ), axial $G d$-TI SE sequences ( $B, F, J, N$, and $R$ ), axial $G d-S W I$ sequences $(C, G, K, O$, and $T)$, TI Gd-Tl MTC sequences $(D, H, L, P$, and $S)$, and a sagittal reformatting Gd-SWI sequence $(U)$. Patient 1 demonstrates an acute juxtacortical/cortical demyelinating lesion in the right precuneus with Gd enhancement in all sequences (orange arrows in $A-D$ ). Patient 2 demonstrates an acute demyelinating plaque on the left corona radiata $(E-F)$. Gd-SWI depicts the central vein within the plaque (arrowhead in G), adding specificity in favor of the demyelinating substrate. Similar to the Gd-TI SE and Gd-TI MTC sequences, the Gd-SWI sequence is also able to demonstrate BBB dysfunction in small lesions (red arrows in I-L). Patient 3 shows an acute demyelinating plaque on the subcortical white matter of the left postcentral gyrus that is only characterized by the Gd-SWI sequence (blue arrows in $M-P$ ). Patient 4 has a venous developmental anomaly in the left semiovale center, surrounded by a parenchymal hyperintensity on a FLAIR sequence $(Q)$. Both the Gd-T1 MTC and Gd-SWI sequences ( $S$ and $T$ ) demonstrate a faint enhancement (arrow in T), which is elegantly demonstrated by the sagittal reformatting Gd-SWI sequence (arrow in $U$ ).

accuracy similar to that of the adopted criterion standard sequence for this purpose and higher than that of the T1 SE sequence, with excellent agreement among the observers in our cohort.

Despite the large number of lesions analyzed, this report has several limitations because it was a single-center retrospective study with a relatively small number of patients without pathologic correlation. Although the visibility of the deep veins was slightly better with the $3 \mathrm{~T}$ scanner, El-Koussy et al ${ }^{18}$ demonstrated similar sensitivity in comparing $1.5 \mathrm{~T}$ with $3 \mathrm{~T}$ scanners. Despite the possibility that the order of the postcontrast sequences could represent a potential limiting bias because the Gd-SWI sequence was acquired first, this reinforces its use as a marker of BBB dysfunction. The use of this new application of the SWI technique requires further study to be incorporated into clinical practice, as well as to implement and standardize the acquisition and interpretation of the results in the context of MS.

\section{CONCLUSIONS}

This study confirmed that the Gd-SWI sequence increased the accuracy of detection of MS-typical contrast-enhancing lesions, including all of their locations and morphologies, similar to Gd-T1 MTC and better than Gd-T1 SE. This new application might add to current knowledge, revealing novel findings of the SWI sequence that are not currently envisioned to have a potential use for clinical practice. Because this study was performed on a 1.5T MR imaging scanner, evaluation of the performance of this sequence at $3 \mathrm{~T}$ would be of interest.

\section{REFERENCES}

1. Ebers GC. Environmental factors and multiple sclerosis. Lancet Neurol 2008;7:268-77 CrossRef Medline

2. Cristiano E, Rojas JI. Multiple sclerosis epidemiology in Latin America: an updated survey. Mult Scler J Exp Transl Clin 2017;3: 2055217317715050 CrossRef Medline

3. Rovira Á, Wattjes MP, Tintoré M, et al; MAGNIMS study group. 
Evidence-based guidelines: MAGNIMS consensus guidelines on the use of MRI in multiple sclerosis-clinical implementation in the diagnostic process. Nat Rev Neurol 2015;11:471-82 CrossRef Medline

4. Sicotte NL. Magnetic resonance imaging in multiple sclerosis: the role of conventional imaging. Neurol Clin 2011;29:343-56 CrossRef Medline

5. Thompson AJ, Banwell BL, Barkhof F, et al. Diagnosis of multiple sclerosis: 2017 revisions of the McDonald criteria. Lancet Neurol 2018;17:162-73 CrossRef Medline

6. Barkhof F. The clinico-radiological paradox in multiple sclerosis revisited. Curr Opin Neurol 2002;15:239-45 CrossRef Medline

7. Wattjes MP, Rovira À, Miller D, et al; MAGNIMS study group. Evidence-based guidelines: MAGNIMS consensus guidelines on the use of MRI in multiple sclerosis- establishing disease prognosis and monitoring patients. Nat Rev Neurol 2015;11:597-606 CrossRef Medline

8. Kermode AG, Thompson AJ, Tofts P, et al. Breakdown of the bloodbrain barrier precedes symptoms and other MRI signs of new lesions in multiple sclerosis. Brain 1990;113:1477-89 CrossRef Medline

9. Poser CM, Brinar VV. The nature of multiple sclerosis. Clin Neurol Neurosurg 2004;106:159-71 CrossRef Medline

10. Di Filippo M, Anderson VM, Altmann DR, et al. Brain atrophy and lesion load measures over 1 year relate to clinical status after 6 years in patients with clinically isolated syndromes. J Neurol Neurosurg Psychiatry 2010;81:204-08 CrossRef Medline

11. Tallantyre EC, Dixon JE, Donaldson I, et al. Ultra-high-field imaging distinguishes MS lesions from asymptomatic white matter lesions. Neurology 2011;76:534-39 CrossRef Medline

12. Sati P, Oh J, Constable RT, et al; NAIMS Cooperative. The central vein sign and its clinical evaluation for the diagnosis of multiple sclerosis: a consensus statement from the North American Imaging in Multiple Sclerosis Cooperative. Nat Rev Neurol 2016;12:714-22 CrossRef Medline

13. Hosseini Z, Matusinec J, Rudko DA, et al. Morphology-specific discrimination between MS white matter lesions and benign white matter hyperintensities using ultra-high-field MRI. AJNR Am J Neuroradiol 2018;39:1473-79 CrossRef Medline

14. Sparacia G, Agnello F, Gambino A, et al. Multiple sclerosis: high prevalence of the "central vein" sign in white matter lesions on susceptibility-weighted images. Neuroradiol J 2018;31:356-61 CrossRef Medline

15. Mittal S, Wu Z, Neelavalli J, et al. Susceptibility-weighted imaging: technical aspects and clinical applications, Part 2. AJNR Am J Neuroradiol 2009;30:232-52 CrossRef Medline

16. Hsu CC, Haacke EM, Heyn CC, et al. The T1 shine through effect on susceptibility weighted imaging: an under recognized phenomenon. Neuroradiology 2018;60:235-37 CrossRef Medline

17. Sehgal V, Delproposto Z, Haacke EM, et al. Clinical applications of neuroimaging with susceptibility-weighted imaging. J Magn Reson Imaging 2005;22:439-50 CrossRef Medline

18. El-Koussy M, Schenk P, Kiefer C, et al. Susceptibility-weighted imaging of the brain: does gadolinium administration matter? Eur J Radiol 2012;81:272-76 CrossRef Medline

19. He J, Grossman RI, Ge Y, et al. Enhancing patterns in multiple sclerosis: evolution and persistence. AJNR Am J Neuroradiol 2001; 22:664-69 Medline

20. Gavra MM, Voumvourakis C, Gouliamos AD, et al. Brain MR postgadolinium contrast in multiple sclerosis: the role of magnetization transfer and image subtraction in detecting more enhancing lesions. Neuroradiology 2004;46:205-10 CrossRef Medline

21. Silver NC, Good CD, Barker GJ, et al. Sensitivity of contrast enhanced MRI in multiple sclerosis: effects of gadolinium dose, magnetization transfer contrast and delayed imaging. Brain 1997;120: 1149-61 CrossRef Medline

22. Bozzao A, Bastianello S, Ferone E, et al. Enhanced and unenhanced MR with magnetization transfer in multiple sclerosis. AJNR Am J Neuroradiol 1996;17:1837-42 Medline

23. Mandolesi G, Gentile A, Musella A, et al. Synaptopathy connects inflammation and neurodegeneration in multiple sclerosis. Nat Rev Neurol 2015;11:711-24 CrossRef Medline

24. Louapre C, Lubetzki C. Neurodegeneration in multiple sclerosis is a process separate from inflammation: yes. Mult Scler 2015;21: 1626-38 CrossRef Medline

25. Pérez-Cerdá F, Sánchez-Gómez MV, Matute C. The link of inflammation and neurodegeneration in progressive multiple sclerosis. Multiple Sclerosis and Demyelinating Disorders 2016:1-9

26. Daumer M, Neuhaus A, Morrissey S, et al. MRI as an outcome in multiple sclerosis clinical trials. Neurology 2009;72:705-11 CrossRef Medline

27. Tillema JM, Hulst HE, Rocca MA, et al; MAGNIMS Study Group. Regional cortical thinning in multiple sclerosis and its relation with cognitive impairment: a multicenter study. Mult Scler 2016;22: 901-09 CrossRef Medline

28. Malayeri AA, Brooks KM, Bryant LH, et al. National Institutes of Health perspective on reports of gadolinium deposition in the brain. J Am Coll Radiol 2016;13:237-41 CrossRef Medline

29. Stojanov DA, Aracki-Trenkic A, Vojinovic S, et al. Increasing signal intensity within the dentate nucleus and globus pallidus on unenhanced T1W magnetic resonance images in patients with relapsingremitting multiple sclerosis: correlation with cumulative dose of a macrocyclic gadolinium-based contrast agent, gadobutrol. Eur Radiol 2016;26:807-15 CrossRef Medline

30. Comabella M, Sastre-Garriga J, Montalban X. Precision medicine in multiple sclerosis: biomarkers for diagnosis, prognosis, and treatment response. Curr Opin Neurol 2016;29:254-62 CrossRef Medline

31. Hiehle JF Jr, Grossman RI, Ramer KN, et al. Magnetization transfer effects in MR-detected multiple sclerosis lesions: comparison with gadolinium-enhanced spin-echo images and nonenhanced T1weighted images. AJNR Am J Neuroradiol 1995;16:69-77 Medline

32. Eichinger P, Kirschke JS, Hoshi MM, et al. Pre- and postcontrast 3D double inversion recovery sequence in multiple sclerosis: a simple and effective MR imaging protocol. AJNR Am J Neuroradiol 2017;38: 1941-45 CrossRef Medline

33. Crombé A, Saranathan M, Ruet A, et al. MS lesions are better detected with 3D T1 gradient-echo than with 2D T1 spin-echo gadolinium-enhanced imaging at 3T. AJNR Am J Neuroradiol 2015;36: 501-07 CrossRef Medline

34. Gupta A, Al-Dasuqi K, Xia F, et al. The use of noncontrast quantitative MRI to detect gadolinium-enhancing multiple sclerosis brain lesions: a systematic review and meta-analysis. AJNR Am J Neuroradiol 2017;38:1317-22 CrossRef Medline

35. Testaverde L, Caporali L, Venditti E, et al. Diffusion tensor imaging applications in multiple sclerosis patients using 3T magnetic resonance: a preliminary study. Eur Radiol 2012;22:990-97 CrossRef Medline

36. Zhang Y, Gauthier SA, Gupta A, et al. Magnetic susceptibility from quantitative susceptibility mapping can differentiate new enhancing from nonenhancing multiple sclerosis lesions without gadolinium injection. AJNR Am J Neuroradiol 2016;37:1794-99 CrossRef Medline

37. Ciccarelli O, Barkhof F, Bodini B, et al. Pathogenesis of multiple sclerosis: insights from molecular and metabolic imaging. Lancet Neurol 2014;13:807-22 CrossRef Medline

38. Rauscher A, Sedlacik J, Barth M, et al. Magnetic susceptibilityweighted MR phase imaging of the human brain. AJNR Am J Neuroradiol 2005;26:736-42 Medline

39. Haacke EM, Mittal S, Wu Z, et al. Susceptibility-weighted imaging: technical aspects and clinical applications, Part 1. AJNR Am J Neuroradiol 2009;30:19-30 CrossRef Medline

40. Mistry N, Abdel-Fahim R, Samaraweera A, et al. Imaging central veins in brain lesions with $3-\mathrm{T} \mathrm{T} 2^{\star}$-weighted magnetic resonance imaging differentiates multiple sclerosis from microangiopathic brain lesions. Mult Scler 2016;22:1289-96 CrossRef Medline

41. Fahrendorf D, Schwindt W, Wölfer J, et al. Benefits of contrast-enhanced SWI in patients with glioblastoma multiforme. Eur Radiol 2013:23:2868-79 CrossRef Medline 